\title{
Hemolymph metagenome of endemic amphipod Eulimnogammarus verrucosus from Lake Baikal
}

\author{
Ekaterina Shchapova \\ Irkutsk State University, Irkutsk, Russia \\ shchapova.katerina@gmail.com \\ Anton Gurkov \\ Irkutsk State University, Irkutsk, Russia \\ a.n.gurkov@gmail.com
}

\author{
Natalia Belkova \\ Scientific Centre for Family Health and \\ Human Reproduction Problems, \\ Irkutsk, 664033 Russia \\ nlbelkova@gmail.com
}

\author{
Renat Adelshin \\ Irkutsk Anti-Plague Research Institute \\ of Siberia and Far East, Irkutsk, Russia; \\ Irkutsk State University, Irkutsk, Russia \\ adelshin@gmail.com \\ Maxim Timofeyev \\ Irkutsk State University, Irkutsk, Russia \\ m.a.timofeyev@gmail.com
}

\begin{abstract}
A diverse number of studies indicates that the hemolymph of aquatic invertebrates, apparently healthy invertebrates, is non-sterile. In this study we for the first time characterized the bacterial diversity in hemolymph of endemic amphipods from Lake Baikal on the example of abundant littoral species Eulimnogammarus verrucosus.
\end{abstract}

Keywords - hemolymph, amphipod, bacteria, Baikal

\section{Motivation and aim}

\section{Motivation}

The aquatic environment contains a diverse and rich bacterial flora and, because of this fact, the presence of microbiota in the hemolymph is mostly reported for aquatic invertebrates [1]. Moreover, some microorganisms not only survive in the animal hemolymph, but also can be beneficial for the host [2]. Certain local microorganisms can compete with incoming ones and stimulate the host immune system, but in some cases, they can also become potential pathogens $[3,4]$. The hemolymph microbiota, therefore, is a factor that must be considered when predicting the impact of negative environmental conditions on invertebrate populations. Crustaceans, in particular diverse endemic amphipods (Amphipoda), are one of the main ecosystem components in pristine Lake Baikal, but their hemolymph microbiomes have never been investigated using modern sequencing techniques. Aim

The aim of the study was to characterize the hemolymph microbiome of one of the most abundant littoral amphipods in Lake Baikal, Eulimnogammarus verrucosus (Gerstfeldt, $1858)$, by $16 \mathrm{~S}$ rDNA amplicon meta-sequencing.

\section{Methods}

DNA was isolated from pooled hemolymph of adult amphipods caught on the Baikal shoreline from two locations separated by the Angara River outflow. The first group of animals was collected in the port Baikal in June of 2016 (2 prepared hemolymph samples, 3 individuals per pool) and the second group was caught in Listvyanka village in September of 2016 ( 2 samples, 4 animals per pool). Amphipods were acclimated to laboratory conditions in aquaria for about a week. The surface of amphipod chitin was sterilized with ethanol before hemolymph extraction. The DNA samples were analyzed using a 454 GS Junior sequencer (Roche) after prior amplification of V4-V6 variable regions of $16 \mathrm{~S}$ rDNA (500F primer:

CCAGCAGCYGCGGTAAN; 1064R primer CGACRRCCATGCANCACCT). The IDTAXA program with the GTDB database was used for taxonomic classification of the obtained amplicon libraries [5].

\section{Results}

Microbiomes of the analyzed hemolymph samples of $E$. verrucosus were generally similar within each of the locations but varied in composition between the locations. In particular, we identified in total 29 bacterial genera, but only five of them were present in all samples: Acinetobacter, Flavobacterium, Arcicella, Polaromonas, and Pseudomonas. As the core components of $E$. verrucosus hemolymph metagenome these genera may deserve the main attention during further research on host-symbiont relationships for amphipods of Lake Baikal.

\section{ACKNOWLEDGMENT}

The study was funded by the RFBR grant \# 19-34-90137.

\section{REFERENCES}

[1] Wang, X. W., \& Wang, J. X. (2015). Crustacean hemolymph microbiota: Endemic, tightly controlled, and utilization expectable. Molecular immunology, 68(2), 404-411.

[2] Desriac, F., Le Chevalier, P., Brillet, B., Leguerinel, I., Thuillier, B., Paillard, C., \& Fleury, Y. (2014). Exploring the hologenome concept in marine bivalvia: haemolymph microbiota as a pertinent source of probiotics for aquaculture. FEMS microbiology letters, 350(1), 107116.

[3] Schmitt, P., Rosa, R. D., Duperthuy, M., de Lorgeril, J., Bachère, E., \& Destoumieux-Garzón, D. (2012). The antimicrobial defense of the Pacific oyster, Crassostrea gigas. How diversity may compensate for scarcity in the regulation of resident/pathogenic microflora. Frontiers in microbiology, $3,160$.

[4] Zhang, X., Sun, Z., Zhang, X., Zhang, M., \& Li, S. (2018). Hemolymph microbiomes of three aquatic invertebrates as revealed by a new cell extraction method. Appl. Environ. Microbiol., 84(8), e02824-17.

[5] Murali, A., Bhargava, A., \& Wright, E. S. (2018). IDTAXA: a novel approach for accurate taxonomic classification of microbiome sequences. Microbiome, 6(1), 1-14. 